\title{
REPRESENTATIONS OF PURE SYMMETRIC AUTOMORPHISM GROUPS OF RAAGS
}

\author{
JAVIER ARAMAYONA \& CONCHITA MARTÍNEZ PÉREZ
}

\begin{abstract}
We study representations of the pure symmetric automorphism group $\operatorname{PAut}\left(A_{\Gamma}\right)$ of a RAAG $A_{\Gamma}$ with defining graph $\Gamma$.

We first construct a homomorphism from $\operatorname{PAut}\left(A_{\Gamma}\right)$ to the direct product of a RAAG and a finite direct product of copies of $F_{2} \times F_{2}$; moreover, the image of $\operatorname{PAut}\left(A_{\Gamma}\right)$ under this homomorphism is surjective onto each factor. As a consequence, we obtain interesting actions of $\operatorname{PAut}\left(A_{\Gamma}\right)$ on non-positively curved spaces

We then exhibit, for connected $\Gamma$, a RAAG which property contains $\operatorname{Inn}\left(A_{\Gamma}\right)$ and embeds as a normal subgroup of $\operatorname{PAut}\left(A_{\Gamma}\right)$. We end with a discussion of the linearity problem for $\operatorname{PAut}\left(A_{\Gamma}\right)$.
\end{abstract}

\section{INTRODUCTION}

Given a group $G$ with a finite generating set $X$, the pure symmetric automorphism group $\operatorname{PAut}(G)$ is the group of those automorphisms of $G$ that send every generator in $X$ to a conjugate of itself. In this paper, we will be interested in representations of pure symmetric automorphism groups of right-angled Artin groups (RAAGs) with respect to the standard generating set; see section 2 for definitions.

An important and motivating example is that of the free group $F_{n}$ on $n$ letters $x_{1}, \ldots, x_{n}$. Humphries [14] proved that PAut $\left(F_{n}\right)$ is generated by the partial conjugations $x_{j} \mapsto x_{i} x_{j} x_{i}^{-1}$. This revealed a strong relation between $\operatorname{PAut}\left(F_{n}\right)$ and braid groups, for Goldsmith [12] had previously showed that the subgroup of $\operatorname{Aut}\left(F_{n}\right)$ generated by partial conjugations is isomorphic to the fundamental group of the configuration space of $n$ unknotted, unlinked circles in $\mathbb{S}^{3}$. Shortly afterwards, McCool [18] gave an explicit presentation of $\operatorname{PAut}\left(F_{n}\right)$ in terms of partial conjugations, where every relation is a commutativity relation between (products of) generators.

For a general RAAG $A_{\Gamma}$, Humpries's result above was extended by Laurence [17], who proved that $\operatorname{PAut}\left(A_{\Gamma}\right)$ is generated by partial conjugations. In addition, Toinet 22 proved that $\operatorname{PAut}\left(A_{\Gamma}\right)$ has a presentation similar in spirit to McCool's presentation of $\operatorname{PAut}\left(F_{n}\right)$. This was subsequently refined by Koban-Piggott [15], who showed that it suffices to consider a finite set of commutation relations between (products of) partial conjugations of $A_{\Gamma}$.

Date: August 29, 2018.

The first named author is partially supported by RYC-2013-13008 and the second one by Gobierno de Aragón and European Regional Development Funds. Both authors were funded by grant MTM2015-67781. 
1.1. Subdirect images of $\operatorname{PAut}\left(A_{\Gamma}\right)$. The first aim of this paper is to use Koban-Piggott's presentation [15] of $\operatorname{PAut}\left(A_{\Gamma}\right)$ in order to produce a (noninjective) homomorphism of PAut $\left(A_{\Gamma}\right)$ to the product of a RAAG times a direct product of copies of $F_{2} \times F_{2}$. Recall that a subgroup of a direct product of groups is said to be a subdirect product if its projection onto each direct factor is surjective. We will show:

Theorem 1.1. For every graph $\Gamma$ there exist a graph $\Delta$, a number $N \geq 0$, and a homomorphism

$$
\operatorname{PAut}\left(A_{\Gamma}\right) \rightarrow A_{\Delta} \times \prod_{i=1}^{N}\left(F_{2} \times F_{2}\right)
$$

whose image is a subdirect product. Moreover, the image of every partial conjugation has infinite order.

In fact, the homomomorphism of Theorem 1.1 is constructed explicitly, and $N$ and $\Delta$ are determined in terms of the graph $\Gamma$. In this direction, following [6] we say that a pair of vertices $v, w$ of $\Gamma$ form a $S I L$-pair if $v \notin \operatorname{lk}(w)$ and $\Gamma \backslash \operatorname{lk}(v) \cap \mathrm{lk}(w)$ has a connected component $Y$ with $v, w \notin Y$. Then the number $N$ in Theorem 1.1 is equal to half the number of SIL-type relations in Koban-Piggott's presentation of $\operatorname{PAut}\left(A_{\Gamma}\right)$ (see the comment after the proof of Theorem 1.1), and $A_{\Delta}$ is the RAAG obtained from PAut $\left(A_{\Gamma}\right)$ by killing every partial conjugation that appears in a SIL-type relation. In the specific case when $A_{\Gamma}=F_{n}$, every partial conjugation appears in a SIL-type relation, and thus we have $\Delta=\varnothing$ and $N=\frac{n(n-1)(n-2)}{2}$.

It is easy to see that the homomorphism given by Theorem 1.1 is not injective unless $\operatorname{PAut}\left(A_{\Gamma}\right)$ is a RAAG itself; see Proposition 3.2 . Hence a natural question is:

Question 1.2. Determine the kernel of the homomorphism in Theorem 1.1.

Applications to $\operatorname{CAT}(0)$-actions. A result of Bridson [3, Theorem 1.1] asserts that, for $n$ sufficiently large, if $\operatorname{Aut}\left(F_{n}\right)$ acts by (semisimple) isometries on a complete $\mathrm{CAT}(0)$ space, then every transvection (and thus every partial conjugation also) is elliptic, i.e. it fixes a point. By considering induced actions, the same result holds for finite-index subgroups of $\operatorname{Aut}\left(F_{n}\right)$ too.

However, the presence of transvections is crucial for these results. Indeed, Koban-Piggott's presentation of $\operatorname{PAut}\left(F_{n}\right)\left[15\right.$ implies that $\operatorname{PAut}\left(F_{n}\right)$ (which recall is generated by all partial conjugations) has non-trivial abelianization, and thus acts by hyperbolic isometries on a CAT(0) space $X$. However, the action so obtained is abelian, in the sense that the image of the homomorphism $\operatorname{PAut}\left(F_{n}\right) \rightarrow \operatorname{Isom}(X)$ is abelian.

In sharp contrast, composing the homomorphism $\rho$ from Theorem 1.1 with a faithful representation of $F_{2}$ into (say) $\operatorname{PSL}(2, \mathbb{Z})$, we obtain the following:

Corollary 1.3. For every $n>0$, PAut $\left(F_{n}\right)$ admits a non-abelian $\operatorname{CAT}(0)$ action where every partial conjugation acts hyperbolically.

1.2. Normal RAAG subgroups of $\operatorname{PAut}\left(A_{\Gamma}\right)$. A well-known corollary of a result of Davis-Januszkiewicz [10] is that RAAGs are linear. As a 
consequence, we may use the homomorphism constructed in Theorem 1.1 to construct a non-faithful linear representation of $\operatorname{PAut}\left(A_{\Gamma}\right)$. In light of the analogy between $\operatorname{PAut}\left(F_{n}\right)$ and braid groups alluded to above, a natural (open) question is whether $\operatorname{PAut}\left(A_{\Gamma}\right)$ is linear. It is easy to see that the group of inner automorphisms $\operatorname{Inn}\left(A_{\Gamma}\right)$ of $A_{\Gamma}$, which is isomorphic to the quotient of $A_{\Gamma}$ by its center, is itself a RAAG that embeds as a normal subgroup of $\operatorname{PAut}\left(A_{\Gamma}\right)$.

In the second part we construct, for connected $\Gamma$, a graph $\hat{\Gamma}$ such that $A_{\hat{\Gamma}}$ properly contains $\operatorname{Inn}\left(A_{\Gamma}\right)$, and it still embeds in $\operatorname{PAut}\left(A_{\Gamma}\right)$ thus providing a big linear normal subgroup of $\operatorname{PAut}\left(A_{\Gamma}\right)$. More concretely, we will show:

Theorem 1.4. Let $\Gamma$ be a connected graph. Then there exists a right-angled Artin group $A_{\hat{\Gamma}}$ and an injective map

$$
A_{\hat{\Gamma}} \rightarrow \operatorname{PAut}\left(A_{\Gamma}\right)
$$

whose image is normal and contains $\operatorname{Inn}\left(A_{\Gamma}\right)$. Moreover if $\Gamma$ has no SIL pair, this map is an isomorphism.

In [7, Section 3], Charney and Vogtmann consider a subgroup $K$ of the outer automorphism group of $A_{\Gamma}$ which is generated by cosets of certain partial conjugations, and which they prove is abelian. As it turns out, a similar reasoning to that of the proof of Theorem 1.4 implies that the lift $\tilde{K}$ of $K$ to $\operatorname{Aut}\left(A_{\Gamma}\right)$ is a RAAG, and that it embeds as a normal subgroup of $\operatorname{PAut}\left(A_{\Gamma}\right)$. However, in general $\tilde{K}$ will be properly contained in the group $A_{\hat{\Gamma}}$ given by Theorem 1.4. See Remark 4.7 for more details.

The plan of the paper is as follows. In section 2 we will give the necessary background on right-angled Artin groups and their automorphism groups. Then in section 3 we proceed to construct the homomorphism of Theorem 1.1. Finally, in section 4 we prove Theorem 1.4 .

Acknowledgements. These ideas sprouted from conversations during the $11^{\text {th }}$ Barcelona Weekend in Group Theory in May 2016. The authors are grateful to the organizer of the conference, Pep Burillo.

\section{Graphs, RAAGs, AND their aUtomorphisms}

In this section we will briefly review the necessary definitions needed for the proofs of our main results.

2.1. Graphs. Throughout, $\Gamma$ will denote a finite simplicial graph. We will write $V(\Gamma)$ and $E(\Gamma)$, respectively, for the set of vertices and edges of $\Gamma$.

Given a vertex $v \in V(\Gamma)$, the link $\operatorname{lk}_{\Gamma}(v)$ of $v$ in $\Gamma$ is the set of those vertices of $\Gamma$ which are connected by an edge to $v$. The star of $v$ is $s t_{\Gamma}(v):=$ $\operatorname{lk}_{\Gamma}(v) \cup\{v\}$. If there is no risk of confusion, we will simply write $\operatorname{lk}(v)$ and $\operatorname{st}(v)$ in order to relax notation.

As mentioned in the introduction, we say that the vertices $v, w$ form $a$ SIL if $v \notin \operatorname{lk}(w)$ and $\Gamma \backslash \operatorname{lk}(v) \cap \operatorname{lk}(w)$ has a connected component which does not contain either $v$ or $w$.

Suppose that $v, w$ are not linked. The following terminology comes from [9] (see also [13]). A connected component of $\Gamma-\operatorname{st}(v)$ that is also a connected component of $\Gamma-\operatorname{st}(w)$ will be called a shared component. The 
unique component of $\Gamma-\operatorname{st}(v)$ that contains $w$ will be called the dominant component of $v$. Finally, a subordinate component of $w$ is any connected component of $\Gamma-\operatorname{st}(w)$ that is contained in the dominant component of $v$. By [9, Lemma 2.1], any connected component of $\Gamma-\operatorname{st}(v)$ is of one of these types; moreover $v$ and $w$ form a SIL if and only if there is some shared component.

2.2. Right-angled Artin groups. Let $\Gamma$ be a finite simplicial graph. The right-angled Artin group (RAAG, for short) defined by $\Gamma$ is the group with presentation

$$
A_{\Gamma}=\langle v \in V(\Gamma) \mid[v, w]=1 \Longleftrightarrow v w \in E(\Gamma)\rangle .
$$

As usual, in order to keep the notation under control, we are blurring the distinction between vertices of $\Gamma$ and the corresponding generators of $A_{\Gamma}$.

Notable examples are the extreme cases when $\Gamma$ has no edges, so that $A_{\Gamma}$ is a free group, and when $\Gamma$ is a complete graph, in which case $A_{\Gamma}$ is a free abelian group.

2.3. Automorphisms of RAAGs. Let $A_{\Gamma}$ the RAAG defined by the graph $\Gamma$, and consider its automorphism group $\operatorname{Aut}\left(A_{\Gamma}\right)$. In the light of the precedent paragraph, for a fixed number $n$ of vertices of $\Gamma$, the group $\operatorname{Aut}\left(A_{\Gamma}\right)$ interpolates between the cases of $\operatorname{Aut}\left(F_{n}\right)$ and $\operatorname{GL}(n, \mathbb{Z})=\operatorname{Aut}\left(\mathbb{Z}^{n}\right)$.

Laurence [17 and Servatius 20 gave an explicit finite set of generators for $\operatorname{Aut}\left(A_{\Gamma}\right)$ for arbitrary $\Gamma$. We now briefly recall these generators:

(i) Graphic automorphisms. These are the elements of $\operatorname{Aut}\left(A_{\Gamma}\right)$ induced by the symmetries of $\Gamma$.

(ii) Inversions. Given $v \in V(\Gamma)$, the inversion on $v$ is the automorphism that maps $v$ to $v^{-1}$ and fixes the rest of generators of $A_{\Gamma}$.

(iii) Transvections. Given vertices $v, w \in V(\Gamma)$ with $\operatorname{lk}(v) \subset \operatorname{st}(w)$, the transvection $t_{v w}$ is the automorphism of $A_{\Gamma}$ given by

$$
\left\{\begin{array}{l}
t_{v w}(v)=v w \\
t_{v w}(z)=z, z \neq v .
\end{array}\right.
$$

(iv) Partial conjugations. Given $v \in V(\Gamma)$ and a connected component $A$ of $\Gamma-\operatorname{st}(v)$, the partial conjugation of $A$ by $v$ is the automorphism $c_{A, v}$ of $A_{\Gamma}$ given by

$$
\left\{\begin{aligned}
c_{A, v}(w) & =v^{-1} w v, w \in A \\
c_{A, v}(z) & =z, z \notin A .
\end{aligned}\right.
$$

Laurence [17 and Servatius [20] proved:

Theorem 2.1 ( $[17,[20])$. Aut $\left(A_{\Gamma}\right)$ is generated by the four types of automorphisms described above. In particular, it is finitely generated.

For the sake of completeness, although it will not be needed here, we remark that Day 8$]$ subsequently proved that $\operatorname{Aut}\left(A_{\Gamma}\right)$ is finitely presented, and gave an explicit presentation. 
2.4. Pure symmetric automorphism groups. We will consider the subgroup $\operatorname{PAut}\left(A_{\Gamma}\right)$ of $\operatorname{Aut}\left(A_{\Gamma}\right)$ consisting of those automorphisms that send every generator of $A_{\Gamma}$ to a conjugate of itself. Laurence proved:

Theorem 2.2 (Laurence 17$]$ ). PAut $\left(A_{\Gamma}\right)$ coincides with the subgroup of $\operatorname{Aut}\left(A_{\Gamma}\right)$ generated by partial conjugations.

As mentioned in the introduction, the above theorem was proved earlier by Humphries 14 in the particular case when $A_{\Gamma}$ is a free group. Observe that a consequence of Theorem 2.1 above is that if $\Gamma$ does not have vertices $v, w$ with $\operatorname{lk}(v) \subset \operatorname{st}(w)$, then $\operatorname{PAut}\left(A_{\Gamma}\right)$ has finite index in $\operatorname{Aut}\left(A_{\Gamma}\right)$, since in this case there are no transvections.

As mentioned in the introduction, McCool [18] gave an explicit presentation of PAut $\left(F_{n}\right)$. We record (an equivalent form of) this presentation next. Let $v_{1}, \ldots, v_{n}$ the standard basis of $F_{n}$, and denote by $c_{i j}$ the automorphism of $F_{n}$ given by conjugating $v_{i}$ by $v_{j}$. We have:

Theorem 2.3 (McCool $[18])$. PAut $\left(F_{n}\right)$ is generated by the set $\left\{c_{i j} \mid 1 \leq\right.$ $i, j \leq n\}$, and a complete set of relations is:

(i) $\left[c_{i j}, c_{k l}\right]=1$, whenever $\{i, j\} \cap\{k, l\}=\varnothing$ or $j=l$, and

(ii) $\left[c_{i j} c_{k j}, c_{i k}\right]=1$ whenever $i, j, k$ are pairwise distinct.

Inspired by the above theorem, and using Day's presentation of $\operatorname{Aut}\left(A_{\Gamma}\right)$ [8], Toinet [22] computed an explicit finite presentation PAut $\left(A_{\Gamma}\right)$. This presentation was subsequently refined by Koban-Piggott [15]; we state their result here, slightly reformulated to suit our purposes:

Theorem 2.4 (Koban-Piggott 15$]$ ). PAut $\left(A_{\Gamma}\right)$ is generated by all partial conjugations $c_{A, v}$, subject to the following relations:

(i) $\left[c_{A, v}, c_{B, w}\right]=1$ if either $v=w$ or $v \in \operatorname{lk}(w)$,

(ii) $\left[c_{A, v}, c_{B, w}\right]=1$ if $v \neq w$ and either $(A \cup\{v\}) \bigcap(B \cup\{w\})=\varnothing$ or $(A \cup\{v\}) \subseteq B$ or $(B \cup\{w\}) \subseteq A$,

(iii) $\left[c_{A, v} c_{B, v}, c_{A, w}\right]=1$ whenever $\Gamma-\operatorname{lk}(v) \cap \operatorname{lk}(w)$ has a connected component $A$ which contains neither $v$ nor $w$, and $w \in B$.

As in [9], it is convenient to reformulate these reations using the terminology introduced at the end of Subsection 2.1. So we have that the partial conjugations $c_{A, v}$ and $c_{B, w}$ commute if and only if either at least one of the connected components $A$ and $B$ is subordinate or both are shared but distinct. And case (iii) happens if $A$ is shared and $B$ dominant (and in this case, the pair of vertices $v, w \in V(\Gamma)$ must form a SIL). We will thus refer to relations of type (iii) as $S I L$-type relations in $\operatorname{PAut}\left(A_{\Gamma}\right)$.

Remark 2.5. Observe that, in the case of $A_{\Gamma}=F_{n}$, the presentations of $\operatorname{PAut}\left(F_{n}\right)$ given by Theorems 2.3 and 2.4 coincide. In the case when $A_{\Gamma}=$ $F_{n}$, any pair of vertices $v_{j}$ and $v_{k}$ form a SIL (for $n \geq 3$ ) with shared components of the form $\left\{v_{i}\right\}$ for any other $i$.

2.5. Arrow diagrams. Next, we present a useful combinatorial manner of visualizing PAut $\left(A_{\Gamma}\right)$, in terms of arrow diagrams, as we now introduce.

Given a finite simplicial graph $\Lambda$, as before we denote its vertex set by $V(\Lambda)$ and its edge set by $E(\Lambda)$. 
Definition 2.6. An arrow diagram is a pair $(\Lambda, \mathcal{A})$, where $\Lambda$ is a finite simplicial graph and $\mathcal{A}$ is a subset of $V(\Lambda) \times E(\Lambda)$. The elements of $\mathcal{A}$ are called arrows. Given an arrow $(v, e) \in \mathcal{A}$, we say that $v$ is its initial vertex. Finally, we call $\Lambda$ the graph underlying the arrow diagram.

The motivation for the name "arrow diagram" is that we may represent an arrow diagram as a graph together with some arrows, see figure 1.

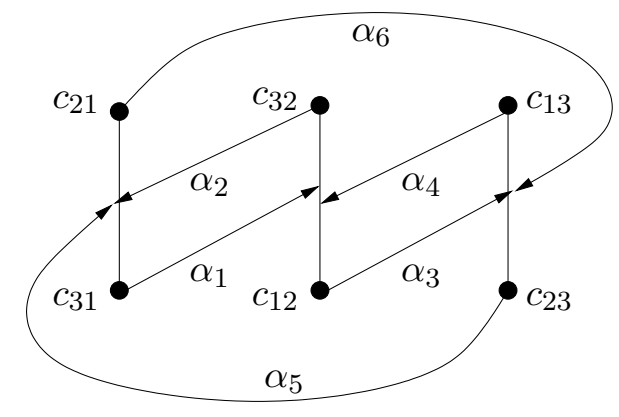

FiguRE 1. The graphical representation of an arrow diagram. In fact, the reader may check from Theorem 2.3 that this is the arrow diagram of $\operatorname{PAut}\left(F_{3}\right)$.

We are now going to define a group from an arrow diagram. We choose to do this is full generality, hoping that the class of groups so defined may be of independent interest.

Definition 2.7. Let $(\Lambda, \mathcal{A})$ be an arrow diagram. The arrow group $G(\Lambda, \mathcal{A})$ defined by $(\Lambda, \mathcal{A})$ is the group generated by the vertices of $\Lambda$, subject to the following relations:

(i) $[v, w]=1$ if and only if $v \in \operatorname{lk}(w)$.

(ii) $[u, v w]=1$ if and only if $v, w$ span an edge $e$ and $(u, e) \in \mathcal{A}$.

Observe that if $\mathcal{A}=\varnothing$, then $G(\Lambda, \mathcal{A})=A_{\Lambda}$, the right-angled Artin group defined by $\Lambda$.

In the light of Theorem 2.4, the following result is immediate:

Proposition 2.8. Let $\Gamma$ be a finite simplicial graph. Then $\operatorname{PAut}\left(A_{\Gamma}\right)$ is an arrow group.

Note that the graph underlying the arrow diagram of $\operatorname{PAut}\left(A_{\Gamma}\right)$ is, in general, not isomorphic to $\Gamma$.

2.5.1. Dual arrows. Viewed as an arrow group, $\operatorname{PAut}\left(A_{\Gamma}\right)$ has a further interesting property, which plays an important rôle in the proof of Theorem 1.1. More concretely, we say that an arrow diagram $(\Lambda, \mathcal{A})$ has property $(D)$ if for every arrow $\alpha=(v, e) \in \mathcal{A}$, there exists a unique arrow $\bar{\alpha}=(\bar{v}, \bar{e}) \in \mathcal{A}$ such that $\bar{v}$ is a vertex of $e, v$ is a vertex of $\bar{e}$, and the subgraph of $\Lambda$ spanned by the vertices of $e, \bar{e}$ has no other edges than $e$ and $\bar{e}$. In this situation, we say that $\alpha$ and $\bar{\alpha}$ are dual arrows. For instance, the arrows $\alpha_{1}$ and $\alpha_{2}$ of Figure 1 are dual to each other. We deduce: 
Proposition 2.9. Let $\Gamma$ be a finite simplicial graph. Then the arrow diagram of $\operatorname{PAut}\left(A_{\Gamma}\right)$ has property $(D)$.

Proof. Consider an arrow $(v, e) \in \mathcal{A}$ in $\operatorname{PAut}\left(A_{\Gamma}\right)$. By Theorem 2.4 it corresponds to a SIL-type relation

$$
\left[c_{A, v} c_{B, v}, c_{A, w}\right]=1,
$$

for $v, w$ a SIL-pair, where $A$ is a shared component and $B$ is dominant thus $w \in B$. Let $C$ be the other dominant component, i.e., the connected component of $\Gamma-\operatorname{lk}(w)$ that contains $v$. Then the partial conjugation $c_{C, w}$ is well-defined and we have

$$
\left[c_{A, w} c_{C, w}, c_{A, v}\right]=1,
$$

as desired. Moreover, the only edges between $c_{A, w}, c_{C, w}, c_{A, v}$ and $c_{B, v}$ are the edges corresponding to $\left[c_{A, w}, c_{C, w}\right]=1$ and $\left[c_{A, v}, c_{B, w}\right]=1$. Thus property (D) holds.

Corollary 2.10. Let $\Gamma$ be a finite simplicial graph. Then the arrow diagram of $\operatorname{PAut}\left(A_{\Gamma}\right)$ has an even number of arrows.

Remark 2.11. Theorem 2.3 implies that the number of arrow pairs in the arrow diagram for $\operatorname{PAut}\left(F_{n}\right)$ is equal to

$$
\frac{n(n-1)(n-2)}{2} \text {. }
$$

Indeed, whenever $\{i, j, k\}$ are pairwise distinct, there is an arrow $\left(c_{i k}, e\left(c_{i j}, c_{k j}\right)\right)$, where $e\left(c_{i j}, c_{k j}\right)$ is the edge between $c_{i j}$ and $c_{k j}$. This arrow has dual arrow $\left(c_{i j}, e\left(c_{j k}, c_{i k}\right)\right)$. Therefore, for each choice of $i \neq j$ we have $(n-2) / 2$ pairs.

\section{Proof of Theorem 1.1}

Before proceeding to proving Theorem 1.1, we need a final piece of notation. Let $\Gamma$ be a finite simplicial graph and $(\Lambda, \mathcal{A})$ the arrow diagram of $\operatorname{PAut}\left(A_{\Gamma}\right)$. We say that a vertex $w \in V(\Gamma)$ is not involved in any arrows if the following two conditions hold

i) there is no arrow of the form $(w, e)$,

ii) there is no arrow of the form $(v, e)$ where $w$ is a vertex of $e$.

Proof of Theorem 1.1. As above, denote by $(\Lambda, \mathcal{A})$ the arrow diagram of $\operatorname{PAut}\left(A_{\Gamma}\right)$. In the light of Corollary 2.10 , we have that $|\mathcal{A}|=2 N$, for some $N \in \mathbb{N}$. We choose an indexing

$$
\mathcal{A}=\left\{\alpha_{1}, \ldots, \alpha_{2 N}\right\}
$$

so that $\alpha_{2 i}=\bar{\alpha}_{2 i-1}$ for all $i=1, \ldots, N$. Now, let $\Delta$ be the subgraph of $\Gamma$ spanned by those vertices of $\Lambda$ that are not involved in any arrows, and $A_{\Delta}$ the associated RAAG. Choose a free basis $a, b$ of $F_{2}$, and write $G$ for the direct product of $N$ copies of $F_{2} \times F_{2}$. We define a map

$$
\rho: V(\Lambda) \rightarrow A_{\Delta} \times G
$$

as follows:

(i) If $v \in V(\Lambda)$ is not involved in any arrows, then we set $\rho(v)=(v, 1)$.

(ii) Otherwise, we set $\rho(v)=(1, g)$, where $g=\left(g_{1}, \ldots, g_{N}\right)$, with the $i$-th entry $g_{i} \in F_{2} \times F_{2}$ being equal to $(1,1)$ unless: 
(a) Suppose $v$ is the initial vertex of the arrow $\alpha_{2 i-1}$ (resp. $\alpha_{2 i}=$ $\left.\bar{\alpha}_{2 i-1}\right)$. In this case, set $g_{i}=(a, 1)$ (resp. $\left.g_{i}=(b, 1)\right)$.

(b) Suppose $v \in \operatorname{lk}(w)$, where $w$ is the initial vertex of the arrow $\alpha_{2 i-1}$, and the edge $e=\{v, w\}$ is the edge of the dual arrow $\bar{\alpha}_{2 i-1}$ (resp. $w$ is the initial vertex of the arrow $\alpha_{2 i}=\bar{\alpha}_{2 i-1}$, and the edge $e=\{v, w\}$ is the edge of the dual arrow $\left.\bar{\alpha}_{2 i}=\alpha_{2 i-1}\right)$. In this case, we set $g_{i}=\left(a^{-1}, a\right)\left(\right.$ resp. $\left.g_{i}=\left(b^{-1}, b\right)\right)$.

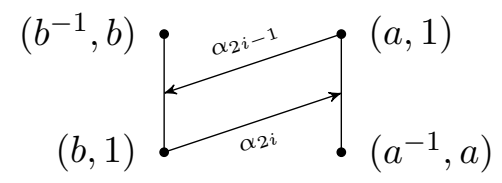

We claim that the map $\rho$ so constructed defines a homomorphism

$$
\rho: G(\Lambda, \mathcal{A})=\operatorname{PAut}\left(A_{\Gamma}\right) \rightarrow A_{\Delta} \times G,
$$

using a slight abuse of notation. In order to do so, we need to verify that $\rho$ preserves the relations of $\operatorname{PAut}\left(A_{\Gamma}\right)$ given in Theorem 2.4. We prove this in two separate claims:

Claim 1. Let $v, w \in V(\Delta)$ be linked vertices. Then

$$
[v, w]=1 \Rightarrow[\rho(v), \rho(w)]=1 .
$$

Proof of Claim 1. First, suppose $v$ is not involved in any arrows, in which case $\rho(v)=(v, 1)$. If $w$ is not involved in any arrows either, then $\rho(w)=$ $(w, 1)$. Otherwise, $\rho(w)=(1, g)$ for some $g \in G$. In either case, $[\rho(v), \rho(w)]=$ 1 and we are done.

Therefore, we have to consider the case when $v$ and $w$ are both involved in some pair of arrows, where by the above it suffices to assume that they are dual to each other. Property (D) implies that, up to interchanging $v$ and $w$, we may suppose that $v$ is the initial vertex of $\alpha_{2 i-1}$ or $\alpha_{2 i}=\bar{\alpha}_{2 i-1}$. Assume, for the sake of concreteness, that we are in the former case, as the other one is totally analogous. In this situation, we have that $\rho(v)=(1, g)$ and $\rho(w)=\left(1, g^{\prime}\right)$, where the $i$-th entry of $g\left(\right.$ resp. $\left.g^{\prime}\right)$ is $(a, 1)\left(\operatorname{resp} .\left(a^{-1}, a\right)\right)$. In particular, $[\rho(v), \rho(w)]=1$, as desired.

Claim 2. For vertices $v, w \in V(\Delta)$,

$$
[u, v w]=1 \Rightarrow[\rho(u), \rho(v) \rho(w)]=1 .
$$

Proof of Claim 2. In this case we know that $v$ and $w$ span an edge $e \in E(\Lambda)$ with $(u, e) \in \mathcal{A}$, so $(u, e)=\alpha_{2 i-1}$ or $\bar{\alpha}_{2 i-1}$ for some $i$. Suppose, again for concreteness, that we are in the former case, so that $u$ is the initial vertex of $\alpha_{2 i-1}$. Up to interchanging $v$ and $w$, we may assume that $v$ is the initial vertex of the arrow $\bar{\alpha}_{2 i-1}$. In particular, we write $\bar{\alpha}_{2 i-1}=(v, \bar{e})$, where $\bar{e} \in E(\Lambda)$ with $u \in \bar{e}$. Let $z$ be the vertex of $\bar{e}$ which is not equal to $u$. From 
the definition of $\rho$, we have:

$$
\begin{aligned}
\rho(u) & =\left(1, g_{u}\right) \\
\rho(v) & =\left(1, g_{v}\right) \\
\rho(w) & =\left(1, g_{w}\right) \\
\rho(z) & =\left(1, g_{z}\right),
\end{aligned}
$$

for some $g_{u}, g_{v}, g_{w}, g_{z} \in G$ whose $i$-th entries are, respectively, equal to $(a, 1)$, $(b, 1),\left(b^{-1}, b\right)$, and $\left(a^{-1}, a\right)$. In particular we see that, when restricted to the $i$-th component of $\rho$, the relation $[\rho(u), \rho(v) \rho(w)]=1$ is preserved. Since the components of $\rho$ correspond precisely to pairs of dual arrows, we obtain the result. This finishes the proof of Claim 2.

In the light of Claims 1 and 2 , we know that $\rho$ defines a homomorphism that we also denote $\rho$

$$
\rho: \operatorname{PAut}\left(A_{\Gamma}\right) \rightarrow A_{\Delta} \times G,
$$

where $G$ is the direct product of $N$ copies of $F_{2} \times F_{2}$. It remains to check that the composition $\rho_{i}$ of $\rho$ with the projection with the $i$-th direct factor of $G$ is surjective, for every $i=1, \ldots, N$. To this end, consider the arrows $\alpha_{2 i-1}$ and $\alpha_{2 i}=\bar{\alpha}_{2 i-1}$. By definition, $\alpha_{2 i-1}$ corresponds to a relation of the form $[u, v w]=1$; thus, up to renaming $v$ and $w, \bar{\alpha}_{2 i-1}$ corresponds to the relation $[w, u z]=1$. Hence, in the $i$-th copy of $F_{2} \times F_{2}$, the entries of $\rho(u)$ and $\rho(w)$ are $(a, 1)$ and $(b, 1)$, and therefore they generate a free group on two generators. Similarly, the entries $\rho(v)$ and $\rho(z)$ are $\left(b^{-1}, b\right)$ and $\left(a^{-1}, a\right)$, respectively. In particular we see that, in this copy of $F_{2} \times F_{2}$, the group generated by $\rho(u), \rho(v), \rho(w)$, and $\rho(z)$ is isomorphic to $F_{2} \times F_{2}$, as desired.

Example 3.1. In the particular case of $\operatorname{PAut}\left(F_{3}\right)$, it is easy to give an explicit description of the generating set under the group homomorphism $\rho$ above. This standard generating set has six elements, and is represented by the arrow diagram of figure 1. In the rest of the example, we will refer to the labelling of that figure. Then one checks that the images of the vertices of $\Lambda$ under $\rho$ are

$$
\begin{aligned}
& \rho\left(c_{21}\right)=\left(a^{-1}, a, 1,1, a, 1\right) \\
& \rho\left(c_{31}\right)=\left(a, 1,1,1, a^{-1}, a\right) \\
& \rho\left(c_{32}\right)=\left(b, 1, a^{-1}, a, 1,1\right) \\
& \rho\left(c_{12}\right)=\left(b^{-1}, b, a, 1,1,1\right) \\
& \rho\left(c_{13}\right)=\left(1,1, b, 1, b^{-1}, b\right) \\
& \rho\left(c_{23}\right)=\left(1,1, b^{-1}, b, b, 1\right) .
\end{aligned}
$$

Observe that $\rho\left(\left[c_{31}, c_{32}\right]\right)=([a, b], 1,1,1,1,1)$ and this element commutes with $\rho\left(c_{13}\right)$. Therefore

$$
\rho\left(\left[\left[c_{31}, c_{32}\right], c_{13}\right]\right)=1
$$

so $\rho$ is not injective.

A souped-up version of this example gives: 
Proposition 3.2. The homomorphism

$$
\rho: \operatorname{PAut}\left(F_{n}\right) \rightarrow \prod_{i=1}^{N} F_{2} \times F_{2}
$$

constructed above is not injective for $n \geq 3$.

Proof. We claim that for any $i, j, k$ pairwise distinct

$$
\rho\left(\left[\left[c_{i j}, c_{i k}\right], c_{j i}\right]\right)=1 .
$$

Consider first $\rho\left(c_{i j}\right)$. Its component on each pair of dual arrows is trivial except in the following cases

$(1)_{s}$ for $1 \leq s \leq n, s \neq i, j$ : arrow from $c_{s j}$ with dual from $c_{s i}$,

$(2)_{s}$ for $1 \leq s \leq n, s \neq i, j$ : arrow from $c_{i j}$ with dual from $c_{i s}$.

Now, doing the same for $\rho\left(c_{i k}\right)$ one sees that for each pair of arrows, one of $\rho\left(c_{i j}\right)$ or $\rho\left(c_{i, k}\right)$ is trivial, except in the case $(2)_{s}$ above for $s=k$. Therefore $\rho\left(\left[c_{i j}, c_{i k}\right]\right)$ is trivial everywhere except for that precise pair of arrows. As $\rho\left(c_{j i}\right)$ is trivial on that pair we get the claim.

Remark 3.3. In fact there is no hope to be able to represent $\operatorname{PAut}\left(F_{n}\right)$ faithfully as a subdirect product of a product of free groups of any rank. Indeed, since $\operatorname{PAut}\left(F_{n}\right)$ is of cohomological type $\mathrm{FP}_{\infty}[2$, a result of BridsonHowie-Miller-Short [4] implies that $\operatorname{PAut}\left(F_{n}\right)$ would be virtually a product of free groups, which is not the case.

\section{Normal RAAG subgroups of $\operatorname{PAut}\left(A_{\Gamma}\right)$}

In this section, we will prove Theorem 1.4. As stated in the introduction, the map of Theorem 1.1 gives a linear representation of $\operatorname{PAut}\left(A_{\Gamma}\right)$ thus we have a linear quotient of this group. In Theorem 1.4 we identify a linear normal subgroup $A_{\hat{\Gamma}}$ of $\operatorname{PAut}\left(A_{\Gamma}\right)$; more precisely a normal subgroup of $\operatorname{PAut}\left(A_{\Gamma}\right)$ which its itself a RAAG. Observe that PAut $\left(A_{\Gamma}\right)$ contains the subgroup $\operatorname{Inn}\left(A_{\Gamma}\right) \cong A_{\Gamma} / Z\left(A_{\Gamma}\right)$ of inner automorphisms as a normal subgroup. Moreover, $\operatorname{Inn}\left(A_{\Gamma}\right)$ is well known to be a RAAG, namely the RAAG associated to the graph $\Gamma-\Lambda$ where $\Lambda$ is the set of vertices of $\Gamma$ which are linked to every vertex other than themselves. There are other ways to find canonical normal subgroups of $\operatorname{PAut}\left(A_{\Gamma}\right)$ which are normal and RAAGs (see [5] and Remark 4.7 below) but our subgroup $A_{\hat{\Gamma}}$ is the biggest possible in a certain sense. For example, PAut $\left(A_{\Gamma}\right)$ is a RAAG itself if and only if there is no SIL [15] and this happens if and only if $A=\operatorname{PAut}\left(A_{\Gamma}\right)$.

As we will see, the RAAG $A_{\hat{\Gamma}}$ will be constructed explicitly via a graph $\hat{\Gamma}$ built from $\Gamma$. Before explaining all this, we need some preliminary work. First, we give the following local characterization of a graph containing no SILs. Given $n \geq 2$ by an $n$-claw we mean the complete bipartite graph $K_{1, n}$. Given an $n$-claw, its unique vertex of valence $n$ is called the center, and the other vertices are called the leaves. We have:

Proposition 4.1. Let $\Gamma$ be a connected graph. The following conditions are equivalent: 
(i) For each 3-claw embedded as full subgraph, there is a labelling $v_{1}, v_{2}, v_{3}$ of the leaves so that $v_{2}$ and $v_{3}$ are connected in $\Gamma-\operatorname{st}\left(v_{1}\right)$ and $v_{1}$ and $v_{2}$ are connected in $\Gamma-\operatorname{st}\left(v_{3}\right)$.

(ii) $\Gamma$ has no SIL.

Proof. We first prove (i) $\Rightarrow$ (ii). Assume, for contradiction, that $\Gamma$ has a SIL pair $v_{1}, v_{2}$. We claim that then $v_{1}$ and $v_{2}$ are leaves of some 3-claw embedded in $\Gamma$ as full subgraph.

In order to see this, we first check that there is a vertex $w$ linked to both $v_{1}$ and $v_{2}$. (We remark that this argument appears in [13, Lemma 6.2], although we include it here for completeness.) Let $C$ be a shared component of the SIL; recall this means that $C$ is both a connected component of $\Gamma-\operatorname{st}\left(v_{1}\right)$ and of $\Gamma-\operatorname{st}\left(v_{2}\right)$. As $\Gamma$ is connected, there is some path in $\Gamma$ connecting $v_{1}$ to $C$. But the fact that $C$ is a connected component of $\Gamma-\operatorname{st}\left(v_{1}\right)$ implies that this path has length 2 ; in particular, there exists some vertex $w$ linked to $v_{1}$ and to some vertex $v_{3}$ in $C$. Now, since $C$ is also a connected component of $\Gamma-\operatorname{st}\left(v_{2}\right)$ we deduce that $w$ is linked to $v_{2}$ too. At this point, we have our embedded 3-claw with center $w$ and leaves $v_{1}, v_{2}$ and $v_{3}$; it remains to check that it is a full subgraph. Observe first that $v_{1}$ and $v_{2}$ cannot be linked in $\Gamma$ by the definition of SIL. Neither can $v_{1}$ be linked to $v_{3}$ because of the choice of $C$; the same applies to $v_{2}$ and $v_{3}$. We claim that (i) cannot hold for the 3 -star formed by $v_{1}, v_{2}, v_{3}, w$. Otherwise, we may assume by symmetry that (say) $v_{2}$ and $v_{3}$ are connected in $\Gamma-\operatorname{st}\left(v_{1}\right)$, which is again impossible by the choice of $C$.

Conversely, assume that (i) fails. Up to relabeling we may assume that there is a full subgraph of $\Gamma$ which is a 3-claw with leaves $v_{1}, v_{2}$ and $v_{3}$, in such way that $v_{1}$ and $v_{2}$ are not connected in $\Gamma-\operatorname{st}\left(v_{3}\right)$ and $v_{1}$ and $v_{3}$ are not connected in $\Gamma-\operatorname{st}\left(v_{2}\right)$. Let $C$ be the connected component of $\Gamma-\operatorname{st}\left(v_{3}\right)$ that contains $v_{1}$. Note that $v_{2} \notin C$, hence $C$ cannot be the dominant component of $\Gamma-\operatorname{st}\left(v_{3}\right)$ with respect to $v_{2}$. Assume that $C$ is a subordinate component; in other words, $C \subseteq L_{2}$ where $L_{2} \subseteq \Gamma-\operatorname{st}\left(v_{2}\right)$ is the dominant component with respect to $v_{3}$. Then, as $v_{1} \in C$ and $v_{3} \in L_{2}$, we would deduce that $v_{1}$ and $v_{3}$ are connected in $\Gamma-\operatorname{st}\left(v_{2}\right)$, which is a contradiction. At this point, $C$ must be a shared component, and thus $v_{2}$ and $v_{3}$ form a SIL pair.

A consequence of the proof of Proposition 4.1 is that, if $\Gamma$ is connected, then for each SIL pair $v, u$ there is a 3-claw with $v$ and $u$ as leaves that fails to satisfy the property in (i). This motivates the following definition.

Definition 4.2. Fix a vertex $v \in \Gamma$, and $w$ a vertex linked to $v$. We say that $w$ is $v$-SIL irrelevant if for any 3-claw $\Delta \subseteq \Gamma$, with center $w$ and $v$ a leaf, and which is a full subgraph of $\Gamma, \Delta$ satisfies condition (i) of Proposition 4.1. In other words, for any full subgraph with form

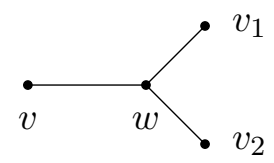

we have (possibly after interchanging $v_{1}$ and $v_{2}$ ) that $v$ and $v_{1}$ are connected in $\Gamma-\operatorname{st}\left(v_{2}\right)$ and at least one of the following holds: either $v_{1}$ and $v_{2}$ are 
connected in $\Gamma-\operatorname{st}(v)$ or $v$ and $v_{2}$ are connected in $\Gamma-\operatorname{st}\left(v_{1}\right)$. The slink of $v$ is now defined as the set of vertices in $\operatorname{lk}(v)$ which are $v$-SIL-irrelevant, that is:

$$
\operatorname{slk}(v)=\{w \in \operatorname{lk}(v) \mid w \text { is } v \text {-SIL irrelevant }\} .
$$

Also, set

$$
\operatorname{sst}(v)=\operatorname{slk}(v) \cup\{v\} .
$$

Note that if a vertex $v$ is not part of any SIL-pair, then $\operatorname{st}(v)=\operatorname{sst}(v)$. In fact, the name "SIL-irrelevant" in the definition above refers to the fact that the removal of $w$ does not create new SIL-type relations (i.e. as in Theorem 2.4(iii)) between the partial conjugations by $v$ in the corresponding connected components (this will be clear below).

Example 4.3. Consider the following graphs
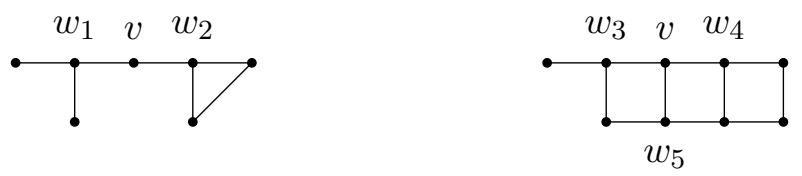

In the first graph, $w_{2}$ is $v$-SIL irrelevant, but $w_{1}$ is not. Thus $\operatorname{sst}(v)=$ $\left\{v, w_{2}\right\}$. In the second graph, $w_{4}$ and $w_{5}$ are both $v$-SIL irrelevant, while $w_{3}$ is not. Hence $\operatorname{sst}(v)=\left\{v, w_{4}, w_{5}\right\}$.

Our next result relates irrelevant components with the connected components of the complement of a SIL:

Lemma 4.4. Let $\Gamma$ be a connected graph, and suppose $u, v$ form a SIL-pair. Let $L_{v}$ be the connected component of $\Gamma-\operatorname{sst}(v)$ that contains $u$. Then, for any connected component $D$ of $\Gamma-\operatorname{st}(v)$ which is either a shared or the dominant component of the $S I L$, we have $D \subseteq L_{v}$. In other words, whenever

(i) either $D$ is also a connected component of $\Gamma-\operatorname{st}(u)$,

(ii) or $u \in D$,

we have $D \subseteq L_{v}$.

Proof. Since $\operatorname{sst}(v) \subseteq \operatorname{st}(v)$ we deduce that if $D$ is the dominant component of $\Gamma-\operatorname{st}(v)$, then $D \subseteq L_{v}$. Now, assume that $D$ is shared. The proof of Proposition 4.1 implies that there exists $w \notin \operatorname{sst}(v)$ which is linked to some vertex in $C$, and also to both $v$ and $u$. As $u, w \in \Gamma-\operatorname{sst}(v)$ and $C \subseteq \Gamma-\operatorname{st}(v) \subseteq \Gamma-\operatorname{sst}(v)$ we deduce $C \subseteq L_{v}$ too.

The graph $\hat{\Gamma}$. We are finally in a position to define the (simplicial) graph $\hat{\Gamma}$ defining the RAAG $A$ which appears in the statement of Theorem 1.4 . The vertices of $\hat{\Gamma}$ are in bijection with pairs $(L, v)$, where $v$ is a vertex of $\Gamma$ and $L$ is a connected component $\Gamma-\operatorname{sst}(v)$. Two such vertices $(L, v)$ and $(T, u)$ are linked in $\hat{\Gamma}$ unless $v$ and $u$ are not linked in $\Gamma, u \in L$ and $v \in T$.

We need one more technical result before proving Theorem 1.4

Lemma 4.5. Let $\Gamma$ be a connected graph, $v$ a vertex of $\Gamma$ and $L \subseteq \Gamma-\operatorname{sst}(v)$ a connected component. Then $L \nsubseteq \operatorname{st}(v)$. 
Proof. Let $w \in L$. First, if $w \notin \operatorname{st}(v)$ we are done. Hence assume that $w \in \operatorname{st}(v)$. Since $w \notin \operatorname{sst}(v)$, we know that $w$ is not $v$-SIL irrelevant. In other words, there exists a 3-claw embedded as a full subgraph of $\Gamma$, with center $w$ and leaves $v, v_{2}$ and $v_{3}$, which fails to satisfy condition of (i) in Proposition 4.1. In particular, $v_{2} \notin \operatorname{st}(v)$ thus $v_{2} \notin \operatorname{sst}(v)$ also but as $v_{2}$ and $w \in L$ are linked, $v_{2} \in L \cap(\Gamma-\operatorname{st}(v))$, as desired.

We are finally in a position to prove Theorem 1.4 .

Proof of Theorem 1.4. We first define $\varphi$ on the vertices of $\hat{\Gamma}$ by the formula

$$
\varphi(L, v)=c_{L, v}
$$

where recall that $c_{L, v}$ is the partial conjugation of $L$ by $v$. We first check that $\varphi$ is well-defined. To this end, it suffices to see that $\varphi$ preserves the standard relators of $A_{\hat{\Gamma}}$. Let $(L, v)$ and $(T, u)$ be two vertices. If either $u=v$ or $u \in \mathrm{lk}_{\Gamma}(v)$, then the partial conjugations $c_{L, v}$ and $c_{T, u}$ commute. So we may assume that $v$ and $u$ are not linked in $\Gamma$ and, say, $v \notin T$. Note that $T \cap(\Gamma-\operatorname{st}(u))=T-(T \cap \operatorname{st}(v))$ is a union of connected components of $\Gamma-\operatorname{st}(v)$, denoted $D_{1}, \ldots, D_{r}$, so that

$$
c_{T, u}=c_{D_{1}, u} \ldots c_{D_{r}, u}
$$

and that none of them is the dominant component of the SIL as $v \notin D_{1} \cup$ $\ldots \cup D_{r}$. Now we distinguish two cases:

(i) Suppose first that $L$ is the connected component of $\Gamma-\operatorname{sst}(v)$ that contains $u$. Then, Proposition 4.4 yields that $L$ contains the dominant and all the shared components of $\Gamma-\operatorname{st}(v)$ with respect to $u$. Now, Theorem 2.4 implies that $\left[c_{L, v}, c_{D_{i}, u}\right]=1$, for $i=1, \ldots, r$. Hence $\left[c_{L, v}, c_{T, u}\right]=1$, as desired.

(ii) Otherwise, $L$ must be a union of shared components, and we are done again by Theorem 2.4 .

At this point, we know that the map $\varphi$ is well-defined. Next, let $\Lambda \subseteq \Gamma$ be the full subgraph whose vertices are linked to every vertex of $\Gamma$ other than themselves. By a result of Servatius [20], the subgroup $A_{\Lambda}$ is precisely the center of $A_{\Gamma}$. Moreover, if $\Gamma_{0}=\Gamma-\Lambda$ then $A_{\Gamma_{0}} \cong A_{\Gamma} / A_{\Lambda}=\operatorname{Inn}\left(A_{\Gamma}\right)$. Thus we have a map

given by the rule

$$
\iota: A_{\Gamma_{0}} \rightarrow A_{\hat{\Gamma}}
$$

$$
v \mapsto \prod\{(L, v) \mid L \text { connected component of } \Gamma-\operatorname{sst}(v)\},
$$

which is obviously well-defined. To see that its image is normal in $A_{\hat{\Gamma}}$ we proceed as follows. Let $u, v$ be vertices of $\Gamma$, and $T$ any connected component of $\gamma-\operatorname{sst}(u)$. If $v \notin T$, then

$$
\iota(v)^{(T, u)}=\iota(v)
$$

Otherwise, if $u \in L$,

$$
(L, v)^{(T, u)}=(L, v)^{\iota(v)},
$$

while if $u \notin L$

$$
(L, v)^{(T, u)}=(L, v) .
$$


In particular,

$$
\iota(v)^{(T, u)}=\iota(v)^{\iota(u)},
$$

as desired.

Now, the maps $\iota$ and $\varphi$ fit in a commutative diagram similar to the one in [6, Page 9]:

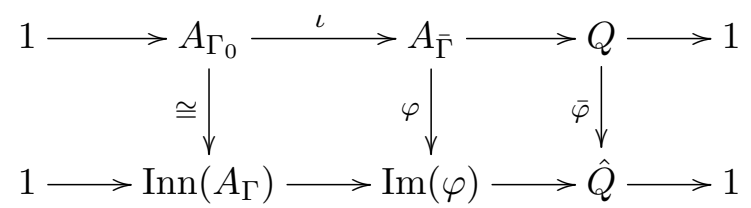

where the injectivity of $\iota$ follows from the commutativity of the diagram, $Q$ and $\hat{Q}$ are the corresponding quotient groups and $\bar{\varphi}$ is the induced map.

We now check that $\operatorname{Im}(\varphi)$ is normal in $\operatorname{PAut}\left(A_{\Gamma}\right)$. Let $u, v$ be vertices of $\Gamma$, $L$ a connected component of $\Gamma-\operatorname{sst}(v)$, and $C$ a connected component of $\Gamma-$ st $(u)$. The same argument used above to show that $\varphi$ is well-defined shows that $\left[c_{L, v}, c_{C, u}\right]=1$, except possibly in the case when $C$ is the dominant component, i.e., when $v \in C$. In this case, let $T$ be the connected component of $\Gamma-\operatorname{sst}(u)$ that contains $C$. Then $T-(T \cap \operatorname{st}(u))=C \cup C_{1} \cup \ldots \cup C_{s}$ for some (non-dominant) components $C_{1}, \ldots, C_{s}$ and we get

$$
\left[c_{L, v}, c_{C_{i}, u}\right]=1
$$

for $i=1, \ldots, s$. As a consequence,

$$
c_{L, v}^{c_{C, u}}=c_{L, v}^{c_{C, u} \prod_{i=1}^{s} c_{C_{i}, u}}=c_{L, v}^{c_{T, u}} \in \operatorname{Im}(\varphi) .
$$

Finally, we claim that the induced map $\bar{\varphi}: Q \rightarrow \hat{Q}$ is injective. We follow the strategy of $[6$. Theorem 3.6], and first show that $Q$ is abelian. To this end, let $(L, v)$ and $(T, u)$ be two vertices of $\hat{\Gamma}$, which are necessarily linked unless $v \in T$. In this case, if $T_{1}, \ldots, T_{r}$ are the remaining connected components of $\Gamma-\operatorname{sst}(u)$, we have $\left[(L, v),\left(T_{i}, u\right)\right]=1$ for $i=1, \ldots, r$, and thus

$$
[(L, v),(T, u)]=\left[(L, v),(T, u) \prod_{i=1}^{r}\left(T_{i}, u\right)\right] \in A_{\Gamma_{0}},
$$

as desired. In fact, it is easy to give an explicit presentation of $Q$, through which one sees that $Q$ is the free abelian group generated by the cosets of the $(L, v)$ 's, after removing exactly one of them for each $v$ (in an arbitrary manner).

To see that $\bar{\varphi}$ is injective, observe that if we had a linear combination $\bar{z}$ of elements such that $\bar{\varphi}(\bar{z})=1$ then any preimage $z \in A_{\hat{\Gamma}}$ would be such that $\varphi(z) \in \operatorname{Inn}\left(A_{\Gamma}\right)$. Let $(L, v)$ be some vertex whose coset appears in $\bar{z}$. As $L \nsubseteq \operatorname{st}(v)$ by Lemma 4.5, $\varphi(z)$ conjugates any $w \in L$ by (at least) $v$. However, it does not conjugate any $u \in T_{v}$ and, using again that $T_{v} \nsubseteq \mathbb{s t}(v)$, we deduce that $\varphi(z)$ cannot be inner.

Remark 4.6. Observe that if $\Gamma$ has no SIL, then $\operatorname{sst}(v)=\operatorname{st}(v)$ for any $v$ thus $\hat{\Gamma}$ has a vertex for each connected component of $\Gamma-\operatorname{st}(v)$ and the group $A_{\hat{\Gamma}}$ is precisely $\operatorname{PAut}\left(A_{\Gamma}\right)$, as shown already in $[6]$. 
Remark 4.7. In [5], in the case when $\Gamma$ is connected and has no triangles, the authors construct a map from a finite index subgroup of Out $\left(A_{\Gamma}\right)$ to a product of groups of the form $\operatorname{Out}(F)$, with $F$ free. The kernel $K$ turns out to be abelian, and is generated by the cosets in $\operatorname{Out}\left(A_{\Gamma}\right)$ of those partial conjugations which are of the form $c_{L, v}$ where $L$ is a connected component of $\Gamma-\{v\}$. This construction was subsequently generalized 7$]$ to arbitrary graphs $\Gamma$.

In general, if we consider the subgroup $N$ of $\operatorname{PAut}\left(A_{\Gamma}\right)$ generated by partial conjugations $c_{L, v}$, with $L$ a connected component of $\Gamma-\{v\}$, one can proceed along similar lines as above to show that $N$ is a RAAG containing $\operatorname{Inn}\left(A_{\Gamma}\right)$ and that it is normal in $\operatorname{PAut}\left(A_{\Gamma}\right)$ and the corresponding quotient is abelian. Moreover, $N$ embeds in $A_{\hat{\Gamma}}$, but in general as a proper subgroup. For example, this is what happens for the graphs of Example 4.3 .

We finish this section with a few words about a possible obstruction to the linearity of $\operatorname{PAut}\left(A_{\Gamma}\right)$ in the case when $\Gamma$ has a SIL. Consider the group $G$ defined as

$$
G=\left(F_{2} \times F_{2}\right) *_{t}
$$

where the stable group is the diagonal group $\Delta\left(F_{2} \times F_{2}\right)$ and $t$ acts as the identity on $\Delta\left(F_{2} \times F_{2}\right)$, i.e., $(g, g)^{t}=(g, g)$. $G$ admits the following explicit presentation:

$$
G=\langle x, a, y, b, t \mid[x, y]=[x, b]=[a, y]=[a, b]=[x y, t]=[a b, t]=1\rangle .
$$

Prassidis and Metaftsis have conjectured that this group is linear. In fact, if $G$ is not linear then the next lemma would imply that $\operatorname{PAut}\left(A_{\Gamma}\right)$ cannot be linear either, provided $\Gamma$ has a SIL:

Lemma 4.8. If $\Gamma$ has a SIL, then $\operatorname{PAut}\left(A_{\Gamma}\right)$ has a subgroup isomorphic to $\left(F_{2} \times F_{2}\right) *_{t}$ where $(g, g)^{t}=(g, g)$.

Proof. Observe that we may express the defining relators of $G$ as

$$
\begin{aligned}
G=\langle x, a, y, b, t \mid[x, y]=[x, b]=[a, y]=[a, b]=[x y, t]=[a b, t]=1\rangle \\
\\
\left\langle x, a, y, b, t \mid a^{y}=a^{b}=a, x^{y}=x^{b}=x, t^{y}=t^{x^{-1}}, t^{b}=t^{a^{-1}}\right\rangle,
\end{aligned}
$$

and that this means that $G$ is the semidirect product of the (normal) subgroup $K$ generated by $x, a$ and $t$, which is free of rank 3 , and a free group of rank 2 generated by $y$ and $b$, where the action is given by partial conjugations.

Now, assume that $\Gamma$ has a SIL pair $v$ and $u$, and let $C$ be a shared component. Choose some vertex $w \in C$ and consider the inner automorphisms $c_{u}$, $c_{v}$ and $c_{w}$ that conjugate by $u, v$ and $w$ respectively. Consider also the partial conjugations $c_{C, v^{-1}}$ and $c_{C, u^{-1}}$, and let $H$ be the subgroup of $\operatorname{PAut}\left(A_{\Gamma}\right)$ generated by these five elements. We claim that the map $f: G \rightarrow H$ induced by

$$
\begin{array}{r}
x \mapsto c_{u}, \\
a \mapsto c_{v}, \\
t \mapsto c_{w}, \\
y \mapsto c_{C, u^{-1}}, \\
b \mapsto c_{C, v^{-1}}
\end{array}
$$


is an isomorphism. To see this, observe that as the vertices $v, u$ and $w$ are pairwise not linked, the subgroup of $A_{\Gamma}$ that they generate is free of rank 3 , and thus the same holds for the conjugations $c_{u}, c_{v}, c_{w}$. Thus the restriction of $f$ yields an isomorphism from $K$ to the subgroup generated by $c_{u}, c_{v}$ and $c_{w}$. The same happens for the restriction of $f$ to the subgroup of $G$ generated by $y$ and $b$ and the subgroup of $H$ generated by $c_{C, u^{-1}}$ and $c_{C, v^{-1}}$. Taking into account the structure of $G$ we deduce that $f$ is in fact an isomorphism.

Remark 4.9. It is not difficult to construct explicit linear representations of $G$. Let $A, B, D, M$ be matrices so that $[A, D]=1=[B, D]$ and $A, B$ generate a free group (we might choose $D=I$ ). Represent the generators of $G$ as follows:

$$
\begin{aligned}
x & \mapsto \operatorname{diag}(A, I) \\
y & \mapsto \operatorname{diag}(I, A) \\
a & \mapsto \operatorname{diag}(B, I) \\
b & \mapsto \operatorname{diag}(I, B) \\
t & \mapsto M \otimes D
\end{aligned}
$$

where $\otimes$ is the Kronecker product. Note that $x y \mapsto \operatorname{diag}(A, A)=I \otimes A$ which commutes with $M \otimes D$.

In light of the above remark, a natural question is:

Question 4.10. Is there is some choice of $A, B, D$ and $M$ so that this representation is faithful?

\section{REFERENCES}

[1] J. Aramayona, C. Martínez-Pérez, On the first cohomology of automorphism groups of graph groups. J. Algebra 452 (2016)

[2] N. Brady, J. McCammond, J. Meier, A. Miller, The Pure Symmetric Automorphisms of a Free Group Form a Duality Group. J. of Algebra 246 (2) (2001).

[3] M. R. Bridson, The rhombic dodecahedron and semisimple actions of $\operatorname{Aut}\left(F_{n}\right)$ on CAT(0) spaces, Fund. Math. 214 (2011).

[4] M. R. Bridson, J. Howie, C. F Miller III, H. Short, Subgroups of direct products of limit groups. Ann. of Math. (2) 170 (2009)

[5] R. Charney, J. Crisp, K. Vogtmann, Automorphisms of 2-dimensional right-angled Artin groups. Geom. Topol. 11 (2007), 2227-2264.

[6] R. Charney, K. Ruane, N. Stambaugh, A. Vijayan, The automorphism group of a graph product with no SIL. Illinois J. Math. 54 (2010),

[7] R. Charney, K. Vogtmann, Finiteness properties of automorphism groups of rightangled Artin groups. Bull. Lond. Math. Soc. 41

[8] M. Day, Peak reduction and finite presentations for automorphism groups of rightangled Artin groups, Geometry $\&$ Topology 13 (2009).

[9] M. Day, R. Wade, Subspace arrangements, BMS invariants and pure symmetric outer automorphisms of right-angled Artin groups. To appear in Group, Geometry and Dynamics.

[10] Michael W. Davis, Tadeus Januszkiewicz. Right-angled Artin groups are commensurable with right-angled Coxeter groups. Journal of Pure and Applied Algebra 153 (2000) 229-235.

[11] E. Formanek, C. Procesi, The automorphism group of a free group is not linear. $J$. Algebra 149 (1992).

[12] D. L. Goldsmith, The theory of motion groups. Michigan Math. J. 28 (1981) 
[13] Mauricio Gutierrez, Adam Piggott and Kim Ruane. On the Automorphisms of a Graph Product of Abelian Groups. Groups Geom. Dyn. 6 (2012), no. 1, 125-153.

[14] S. P. Humphries, On weakly distinguished bases and free generating sets of free groups. Quart. J. Math. Oxford Ser. (2) 36 (1985),

[15] N. Koban, A. Piggott, The Bieri-Neumann-Strebel invariant of the pure symmetric automorphisms of a right-angled Artin group. Illinois J. Math. 58 (2014),

[16] M. R. Laurence. Automorphisms of graph products of groups. PhD thesis, Queen Mary College, University of London, 1993.

[17] M. R. Laurence. A generating set for the automorphism group of a graph group. J. London Math. Soc. (2), 52(2): 318-334, 1995.

[18] J. McCool, On basis-conjugating automorphisms of free groups. Canad. J. Math. 38 (1986).

[19] B. Mühlherr. Automorphisms of graph-universal Coxeter groups. J. Algebra 200(2) 1998.

[20] H. Servatius, Automorphisms of graph groups, J. Algebra 126 (1989).

[21] Jaques Tits, Sur le groupe des automorphismes de certains groupes de Coxeter, Journal of Algebra, 113, no. 2 (1988).

[22] E. Toinet, A finitely presented subgroup of the automorphism group of a right-angled Artin group. J. Group Theory 15 (2012) 